\title{
Unach
}

\section{CULTURA INVESTIGATIVA EN LOS ESTUDIOS DE POSTGRADO DESDE EL ENFOQUE INTEGRADOR TRANSCOMPLEJO}

INVESTIGATIVE CULTURE IN POSTGRADUATE STUDIES FROM THE TRANSCOMPLEJO INTEGRATOR APPROACH

\section{RESUMEN}

La cultura investigativa de una universidad se desarrolla mediante el proceso educativo, la extensión, la vinculación con el entorno y la investigación, que se ejecuta con la intervención de los actores intra y extra institucionales: directivos, docentes, estudiantes y miembros de la comunidad o actores comunitarios. En tal sentido, el propósito del artículo es caracterizar la cultura investigativa en los estudios de postgrado de la Universidad Bicentenaria de Aragua, Venezuela, producto de una investigación fundamentada en el enfoque integrador transcomplejo, con base a fuentes mixtas. La información cuantitativa se recogió mediante realizó la observación de 33 trabajos de investigación y la aplicación de una escala de estimación a 40 docentes de postgrado. A efecto, de la información cualitativa, se realizaron entrevistas a 8 informantes (estudiantes y docentes) de postgrado de la misma universidad. Se concluye que en la cultura investigativa se advierten signos positivos de avance, no obstante, se requiere potenciar las acciones formativas en relación a los docentes que actúan como tutores; así como el posicionamiento del enfoque integrador transcomplejo, como postura investigativa institucional.

Palabras clave: cultura investigativa; enfoque integrador transcomplejo; estudios de postgrado.

\begin{abstract}
The research culture of a university isdeveloped through the educational process, the extension, the link with the context and the research that is carried out with the intervention of intra and extra institutional actors (managers, teachers, students and community members or actors). In this sense, the purpose of the article is to characterize the research culture in the postgraduate studies of a Venezuelan private university, product of a research based on the transcomplejo integrative approach, based on mixed sources. The observation of 33 research works and the application of an estimation scale to 40 postgraduate teachers in order to obtain qualitative information, eight informants (students and teachers) of the same university were interviewed. In the research culture there are positive signs of progress, however, it is necessary to strengthen the training actions in relation to the teachers who act as tutors; as well as the positioning of the transcomplex integrative approach, as an institutional investigative position.
\end{abstract}

Keywords: investigative culture; postgraduate studies; transcomplex integrative approach.
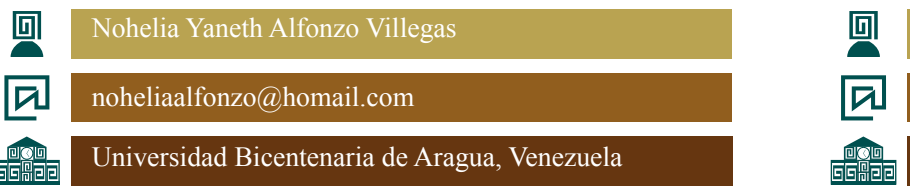


\section{INTRODUCCIÓN}

La cultura investigativa de acuerdo a Martins (2005:55):

...está compuesta por un conjunto de valores, creencias y conceptos básicos, rituales y ceremonias (rutinas programadas y sistemáticas de la cotidianidad de la investigación) y normas (formales e informales) compartidas por el conjunto de individuos (docentes, coordinadores y autoridades) que conforman una manera propia de hacer investigación en un determinado contexto.

De ahí que cada Universidad posee una cultura de investigación particular, que aun cuando no se puede percibir desde afuera, se encuentra en todos sus componentes, puede ser muy fuerte, cohesiva o débil. Su rol es el de guiar el comportamiento, orientando a estudiantes y profesores a realizar satisfactoriamente la investigación, al ofrecerle un sistema de reglas que pueden ser captadas por todos.

En la Universidad Bicentenaria de Aragua (UBA), creada en 1986, con 19 Programas de Postgrado distribuido en siete especializaciones, 11 maestrías y un doctorado, en las áreas de educación, gerencia, derecho, ingeniería y ambiente; se promueve la cultura investigativa, según lo establecido en las Políticas de Investigación (Universidad Bicentenaria de Aragua 2015a), mediante actividades formativas en el área, la asistencia a eventos, realización de investigaciones a lo largo de sus estudios, mediante la posibilidad de realizar publicaciones periódicas, así como a través de la divulgación de la reglamentación y lineamientos propios, tales como el Manual de Trabajo de Grado y Tesis Doctorales, cuya última revisión se hizo en mayo 2017 (Universidad Bicentenaria de
Aragua 2017a) y Orientaciones para la Elaboración de Artículos Académicos (Universidad Bicentenaria de Aragua 2017b), entre otros.

Así mismo, la universidad ha venido desarrollando desde el año 2003 una propuesta investigativa denominada el Enfoque Investigador Transcomplejo, asumido por sus principales proponentes: Villegas, Schavino, Rodríguez, Moreno, Extraño, Reyes, Salazar, Ferrer, Díaz, Lamardo, y Guzmán (2006), como una nueva cosmovisión investigativa de complementariedad metodológica, que puede potenciar los avances y tratar de reducir las limitaciones que presentan las aproximaciones cuantitativas y cualitativas por separado.

\section{El Consejo Universitario (Universidad} Bicentenaria de Aragua 2015b), aprueba desarrollar un modelo educativo con enfoque curricular por competencias transcomplejo, que a constituirse, a largo plazo, en una nueva práctica educativa y de investigación comprometida y colectiva que produzca conocimiento situado y pertinente para la vida.

No obstante, aun cuando la investigación es la finalidad principal de los estudios de postgrado, generalmente los estudiantes la realizan sólo para cumplir con el trabajo de grado o tesis doctoral. Lo planteado se evidencia en la poca e incipiente publicación de los resultados de las investigaciones que se logran concluir. De acuerdo a Morlés, Núñez y Álvarez (2010), la investigación "es escasa, de carácter unidisciplinaria, individualista y de limitado alcance".

Otros autores señalan que los profesores y estudiantes que realizan investigaciones generalmente caen en la tentación de recurrir a figuras dualistas $y$ suelen formularlas como cuantitativas o cualitativas, positivis- 
tas o fenomenológicas, simples o complejas. Este pensamiento que separa, dicotomizado, es el que sigue dominando el acto de investigar en la universidad (Bracho, Molina, Carruyo y Ureña 2010).

Al respecto, Leal (2005:124) afirma que, "la tendencia a rechazar todo lo que no encaja dentro de nuestra racionalidad, le hace mucho daño al desarrollo de la investigación en las universidades". Este tipo de pensamiento está profundamente anclado en la estructura de las operaciones mentales que se realizan en todos los ámbitos de la vida, por ello es difícil su superación.

Tal situación hace que los estudiantes de postgrado en las pocas investigaciones que realizan, se preocupen más por responder a los requerimientos normativos, que a la profundidad de la temática; de ahí que cuando se le presenta otra alternativa para realizar investigaciones, como ocurre en la UBA, el estudiante manifiesta resistencia al cambio por temor a no cumplir las normativas e incorporar modificaciones en la en la práctica investigativa tradicional.

Particularmente, en la Universidad Bicentenaria de Aragua predomina una cierta visión de la investigación basada en lineamientos predeterminados y preconcebidos tendentes a una investigación repetitiva tanto en las temáticas como en la metodología. A tales efectos, un diagnóstico realizado por Moreno (2005) plantea que en la UBA, la mayoría de los trabajos de maestría son de tendencia cuantitativa y a nivel de doctorado predomina lo cualitativo.

Otra particularidad de la praxis investigativa en la universidad estudiada, es que se hace preeminente en problemas cognitivos, casi siempre descontextualizada de los proble- mas sociales. Por su parte, el aprender a investigar se intenta mediante los denominados seminarios de investigación en los programas de especialización, maestría y de doctorado, que en la práctica se han transformado en espacios de realizaciones mecánicas, donde en muchos casos, los participantes construyen el proyecto de investigación, pero no internalizan lo que han hecho.

La situación planteada si bien no es nueva y la Universidad ha realizado acciones para transformarla, mantiene vigencia debido a la necesidad de que la investigación responda a los requerimientos actuales de la sociedad. De ahí que, resulta pertinente caracterizar la cultura investigativa de la Universidad Bicentenaria de Aragua y conocer si se ha posicionado el enfoque integrador transcomplejo, como propuesta institucional para la práctica de la investigación científica.

\section{METODOLOGÍA}

La metodología empleada se apoyó en el enfoque integrador transcomplejo, al respecto Zaa $(2015: 11)$ plantea que:

Es vital entender la transcomplejidad como un ejercicio de pensamiento mediante el cual se crean espacios de reflexión donde se entrelazan las percepciones objetivas y subjetivas de la realidad, sus dimensiones lineales y multidimensionales, las concepciones universales y multiversales, lo aparencial y lo substancial, la naturaleza y la artificialeza, lo esencial y lo existencial, lo inductivo concreto y lo deductivo abstracto, lo material y lo energético; en fin lo racional y lo vivencial; como un intento de conformación de novedosos y variados paisajes cognitivos, que contribuyan a la construcción de inéditos e inexplorados caminos del conocimiento humano... 
La aplicabilidad de este enfoque requiere de multimétodos, trabajo en equipo como proceso de intercolaboración y la reflexibilidad como eje que lo trasciende (Villegas et al. 2006).

La intercolaboración asumida como una nueva forma de entender el trabajo en equipo, donde exista un diálogo continuo entre todas las personas que están participando, implica interacción e intercambio con aquellos que mantienen posiciones y epistemes antagónicos a fin de complementar la percepción de la realidad.

La reflexibilidad, por su parte, implica que el sujeto piensa sus pensamientos, realiza una reflexión sobre sus reflexiones. Se produce por la necesidad de ser consciente de las propias ideas y conductas porque es posible que sean erróneas o que haya otras mejores, es una forma de pensamiento crítico, pues al meditar sobre unos conceptos surge la duda acerca de estos.

En correspondencia con el enfoque integrador transcomplejo asumido, el itinerario metodológico depende de cada investigación. Así para Najmanovich (2001) es construir itinerarios según las problemáticas particulares que se presentan en cada investigación específica. En este caso se utilizan diferentes métodos, pero se le da preeminencia a lo cualitativo por cuanto de acuerdo a Balza (2013) "fundamentalmente debe nutrirse de la reflexión hermenéutica profunda y la dialógica recursiva argumental".

El escenario de esta investigación, es la UBA, específicamente el Decanato de Investigación, Extensión y Postgrado, del cual se extrajo la información requerida de los distintos documentos y actores involucrados en la cultura de investigación: directivos, docentes, tutores, jurados y estudiantes de postgrado.

En la primera fase se utilizó el método analítico para estudiar una muestra de siete
Trabajos Especiales de Grado de Especialización, 21 Trabajos de Grado de Maestría y cinco de Tesis Doctorales. Se hizo utilizando una matriz de revisión documental, construida tomando como criterio el paradigma y el método explícitamente señalado en el texto, así como la línea de investigación institucional en la cual se ubica el trabajo según la temática, también indicada por el autor en cada trabajo.

Esta actividad trato de clarificar cuál es el enfoque epistemológico y método predominante en los trabajos de investigación que se revisaron, para contrastar con el estudio realizado, en la misma universidad, por Moreno (2005), así como para constatar el avance en cuanto al posicionamiento del enfoque integrador transcomplejo. También para establecer el conocimiento que tienen los estudiantes acerca de las líneas de investigación evidenciada en los trabajos revisados (la universidad tiene líneas de investigación institucionales y en correspondencia, líneas de investigación propias del doctorado).

Seguidamente se hizo un estudio de campo, de nivel descriptivo para caracterizar la cultura investigativa de los actores académicos de los estudios de postgrado de la Universidad Bicentenaria de Aragua. A tal efecto se elaboró la Escala CIUBA de elaboración propia, con un total de 50 ítemes con cuatro alternativas de respuestas. Para su elaboración fue necesario operacionalizar la variable estudiada, como se muestra en la tabla 1, a continuación.

La validez de la escala elaborada se hizo mediante el juicio de tres expertos (Doctores en Ciencias de la Educación de tres universidades públicas de la región), el cual evidenció que cinco ítemes generaban confusión en la respuesta. Se asumió la observación mejorando la redacción de los ítems señalados. 
Tabla 1: Operacionalización de la variable investigada

\begin{tabular}{|l|l|l|c|}
\hline \multicolumn{1}{|c|}{ Variable } & \multicolumn{1}{|c|}{ Dimensiones } & Indicadores & Items \\
\hline \multirow{4}{*}{$\begin{array}{l}\text { Cultura } \\
\text { Investigativa }\end{array}$} & $\begin{array}{l}\text { Desempeño } \\
\text { investigativo de los actores } \\
\text { académicos }\end{array}$ & Estudiantes & $1-9$ \\
\cline { 3 - 4 } & \multirow{2}{*}{\begin{tabular}{l} 
Estrategias \\
\cline { 2 - 3 }
\end{tabular}} & Docentes & $10-30$ \\
\cline { 3 - 4 } & & Docentes & $10-30$ \\
\cline { 3 - 4 } & & Promoción & $31-33$ \\
\cline { 3 - 4 } & & Formación & $34-36$ \\
\cline { 3 - 4 } & & Infraestructura de Apoyo & $37-46$ \\
\cline { 3 - 4 } & & Líneas de Investigación & $47-50$ \\
\hline
\end{tabular}

Fuente: elaboración propia.

Incorporadas las observaciones se aplicó el instrumento, en un estudio piloto a un grupo de diez profesores de postgrado, no perteneciente a la muestra definitiva. A los resultados obtenidos se aplicó la fórmula de Alfa de Crombach para determinar la confiabilidad en instrumentos con cuatro alternativas de respuesta, se obtuvo un coeficiente de confiabilidad de 0,93 lo que evidencia una confiabilidad alta, según lo planteado por Ramírez, T. (2007).

El instrumento se aplicó a la totalidad de 40 profesores de postgrado que asistieron a una reunión convocada por las investigadoras para tal fin, siendo el criterio de selección: el deseo de participar, manifestado en la asistencia a la convocatoria. Lo planteado se fundamenta en el principio de intercolaboración asumido en el enfoque integrador transcomplejo. La información recogida se tabuló mediante el análisis porcentual y sus resultados se organizaron mediante cuadros y gráficos en función de la variable estudiada.

El tercer método utilizado fue el estudio de caso cualitativo que es un método que ha ido ganando un gran interés, dadas las posibilidades que presenta en la explicación de nuevos fenómenos. A tales fines se seleccionaron de manera intencional dos grupos de discusión, que para efectos del estudio se denominaron cibercomunidades de investigación, por cuanto se estableció con ellos una conversación mediante internet, a lo cual muchos no respondieron. Esto implico reestructurar una y otra vez los grupos de informantes.

González (2007), expresa al respecto, que el conocimiento producido en grupos grandes se apoya en los mismos principios epistemológicos del estudio de casos, es decir, no es el tamaño del grupo el que define el procedimiento de construcción del conocimiento, sino las exigencias de información que caracterizan la investigación. En este caso los grupos definitivos fueron los siguientes:

\section{Grupo 1: Directivos, Docentes, Tutores y Jurados}

En este caso se seleccionaron cinco directivos, tres docentes, tres tutores y tres jurados, en los diferentes niveles de postgrado: Especialización, Maestría y Doctorado. Sólo cuatro personas aceptaron participar, ventajosamente, los cuatro cumplen los tres roles (docente, tutor y jurado en los tres niveles). Los criterios de selección fueron su disposición para participar en la investigación y ser de diferentes disciplinas, lo cual se cumplió 
ya que una es abogada, otra administradora, una ingeniera y la otra licenciada en educación.

\section{Grupo 2: Estudiantes y Egresados}

En este caso se seleccionaron dos estudiantes en proceso de culminación del postgrado en los programas de Especialización, Maestría y Doctorado para un total de seis personas, sólo cuatro aceptaron participar. En este caso, dos, además de ser estudiantes del doctorado son docentes y tutoras en pregrado y en los programas de especialización y maestrías de la institución.

Se utilizó como técnica, la entrevista tipo conversación, siendo el instrumento en correspondencia el guion de preguntas, para el cual se elaboraron una serie de planteamientos no estructurados, por cuanto sólo contenían preguntas activadoras del proceso comunicacional.

Al respecto González (2007:32) señala que las conversaciones generan una corresponsabilidad que hace a cada uno de los participantes sentirse sujetos del proceso, lo que facilita la expresión particular a través de sus necesidades e intereses. Cada miembro participa de la conversación en forma reflexiva, colaborando a través de las posiciones que asume sobre el tema que lo ocupa. En esta fase la información se recopiló e interpretó simultáneamente, con base en el método hermenéutico.

Las técnicas de interpretación fueron la categorización y estructuración. El proceso de categorización, constituye en una parte fundamental para el análisis e interpretación de los resultados, ya que busca fraccionar la información en subconjuntos y asignarle nombres. Bonilla-Castro y Rodríguez (2005), señalan que es un proceso cognitivo complejo de clasificación según la similitud y diferencias encontradas, a partir de crite- rios previamente establecidos.

De tal manera, que una vez reducidas las entrevistas a categorías, se elaboraron las matrices de categorización, donde el tema central del estudio, se estructuró en categorías y subcategorías. Con todo este sistema de matrices se organizó todo el material recabado, donde se reflejó las expresiones más resaltantes y que se consideraron más pertinentes al estudio. Luego, se elaboró la matriz general de categorías y subcategorías.

Paralelamente se realiza el proceso de estructuración asumido como integración de categorías específicas en categorías más generales por cada informante. Implico elaborar la síntesis descriptiva, el esquema organizacional, los nexos y relaciones, lo que se logra interpretando y explicando las categorías con la propia información. Permite integrar mediante flechas y diseños gráficos las distintas categorías en formas más complejas.

\section{RESULTADOS Y DISCUSIÓN}

Los resultados obtenidos se muestran a continuación, organizados en tablas y figuras, según el tipo de información obtenida. En la tabla 2 se aprecian los resultados de la observación documental.

La revisión de la matriz permite evidenciar que de los 33 trabajos de investigación revisados, 78,7\% corresponden al enfoque positivista, $18,1 \%$ al interpretativo y $3,2 \%$ al complejo, lo cual evidencia que la investigación sigue siendo tradicional, disciplinaria y reduccionista. En este aspecto vale destacar que se hicieron más observaciones de las reportadas, especialmente a nivel doctoral por considerar que es tal vez donde se podría haber empezado a utilizar la propuesta transcompleja. Sin embargo, se encontró sólo una que utilizo el enfoque dialógico complejo. 
Al respecto, Alfonzo (2015), plantea la importancia de que la investigación doctoral sea realizada desde un pensamiento que emplee como episteme el enfoque integrador transcomplejo, ya que las tesis doctorales deben caracterizarse por su originalidad, es decir, deben proponer aspectos no abordados antes por ningún otro investigador, lo cual garantiza la transcomplejidad por cuanto plantea una mirada desde multiples disciplinas y actores, más abarcadora y profunda.
En cuanto al método, se evidenciaron que de los 33 trabajos revisados, $96,9 \%$ trabajos emplearon el método único, de los cuales $54,5 \%$ fueron analíticos, de los cuales el $18,2 \%$ en especialidad y $36,3 \%$ en maestría, $24,2 \%$ descriptivo en maestría, $15,1 \%$ hermenéutico de los cuales el $12,1 \%$ en doctorado y $3 \%$ en especialidad y $3 \%$ etnográfico en maestría y un solo caso de métodos mixtos en doctorado. Así de acuerdo con Alfonzo (2015:10) "en la práctica investigativa se produce una hegemonía que busca

Tabla 2: Resultados de la Observación Documental

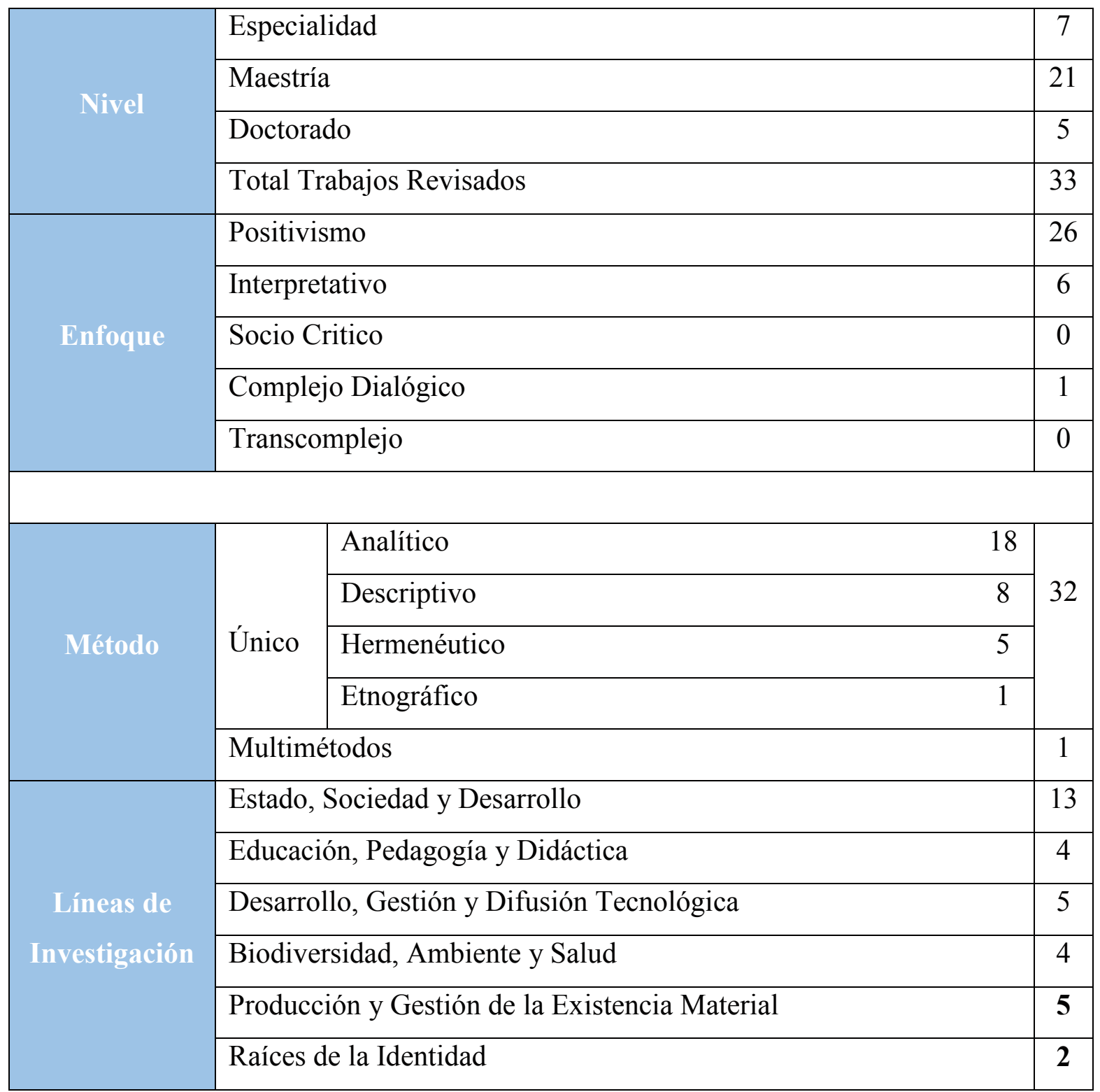

Fuente: elaboración propia. 
gobernar la investigación de manera unimétodica", producto del no claro manejo de los métodos que sustentan los enfoques paradigmáticos.

En cuanto a las líneas de investigación se evidencia el empleo con preeminencia de la línea Estado, Sociedad y Desarrollo (39,3\%), seguida de Desarrollo, Gestión y Difusión Tecnológica $(15,1 \%)$ y Producción y Gestión de la Existencia Material (15,1\%), subsiguientemente, la línea Biodiversidad, Ambiente y Salud $(12,1 \%)$ y en último lugar la línea Raíces de la Identidad (6,3\%).

En relación a las líneas de investigación del Doctorado en Ciencias de la Educación, en la revisión realizada se observó únicamente el empleo de la línea Educación, Pedagogía y Didáctica (80\%), pues de las cinco tesis doctorales revisadas, una de ellas, hace mención de la línea institucional Desarrollo, Gestión y Difusión Tecnológica. Esto hace suponer, que los actores académicos desconocen la existencia del resto de las líneas del Doctorado.

Resultados de Aplicación de la Escala CIUBA

Los resultados obtenidos para la variable cultura investigativa medida mediante la escala CIUBA, se presentan en las tablas 3 a la 7 , estructurados según dos dimensiones: desempeño investigativo de los actores académicos y estrategias utilizadas para promover la investigación. En el caso del desempeño, se presenta separada la información aportada por los docentes respecto a los estudiantes y acerca de si mismos.

Los resultados favorables obtenido para el indicador estudiantes manifiesta que su desempeño investigativo presenta mayor fortaleza en el uso de las TIC ya que el 100\% de estos la utilizan para la investigación, según los docentes encuestados; el $80 \%$ actúa como un investigador activo y participa en la promoción de investigación, el 70\%

Tabla 3: Indicador Estudiantes

\begin{tabular}{|l|c|}
\hline \multirow{2}{*}{\multicolumn{1}{|c|}{ Items }} & FAV \\
\cline { 2 - 2 } & $\%$ \\
\hline Apoyo & 60 \\
\hline Promoción & 80 \\
\hline Asistencia & 20 \\
\hline Información & 70 \\
\hline Investigador activo & 80 \\
\hline Uso TIC & 100 \\
\hline Comunicación con pares & 60 \\
\hline Promedio \% & $67,1 \%$ \\
\hline
\end{tabular}

Fuente: elaboración propia. 
tiene conocimiento de las TIC, el 60\% recibe apoyo en la realización de sus investigaciones y sólo el $20 \%$ asiste a actividades relacionadas con la investigación como reunión de líneas de investigación y/o jornadas.

Tales porcentajes permiten inferir que se percibe que los estudiantes no reciben suficiente apoyo para investigar y no son sistemáticos en la asistencia a actividades fuera del aula, asumiendo que el cursar estudios de postgrado se limita a asistir a clases.

Los resultados favorables obtenidos para el indicador docentes, que se muestran en la tabla 4, evidencias que presentan fortaleza, pues el $80 \%$ se declaran comprometidos a investigar y a publicar, asumen como investigadores activos y asisten a eventos nacionales. El $60 \%$ se encuentran informados, manejo las TIC en la búsqueda de la información, se comunican con sus pares y sólo el $20 \%$ asisten a eventos internacionales, siendo este indicador la mayor debilidad. Lo planteado está directamente relacionado con la situación país, especialmente, con el control de divisas y con la débil infraestruc- tura de apoyo, ya mencionada en los indicadores anteriores.

El promedio favorable obtenido $(66,3 \%)$ para ambos actores en su desempeño como investigadores evidencia debilidades particularmente en la asistencia a eventos investigativos.

La tabla 5 muestra los resultados favorables para la dimensión estrategias, donde se evidencia que la mayor fortaleza es la formación ya que el $100 \%$ declara poseer información en el área, el 78\% reconocen que en la universidad existen de líneas de investigación; el $63,4 \%$ considera que se realizan actividades de promoción de la investigación en la universidad y que participan en estas; sólo el $31 \%$ de los docentes consideran que la infraestructura de apoyo a la investigación que tiene la universidad es adecuada, siendo esta la mayor debilidad.

\section{Al respecto la Organización de las Naciones Unidas para la Educación, la Ciencia y la Cultura (UNESCO) afirma que:}

Las estrategias para promover la investiga-

Tabla 4: Indicador Docentes

\begin{tabular}{|l|c|}
\hline \multirow{2}{*}{\multicolumn{1}{|c|}{ Ittems }} & FA V \\
\cline { 2 - 2 } & $\%$ \\
\hline Informados & 60 \\
\hline Comprometidos a investigar & 80 \\
\hline Comprometidos a publicar & 80 \\
\hline Manejo de las TIC & 60 \\
\hline Comunicación con sus pares & 60 \\
\hline Investigadores activos & 80 \\
\hline Asistencia a eventos & 40 \\
\hline Promedio \% & 65,5 \\
\hline
\end{tabular}

Fuente: elaboración propia. 
Tabla 5: Dimensión Estrategias

\begin{tabular}{|l|c|}
\hline \multicolumn{1}{|c|}{ Indicadores } & $\%$ \\
& Favorable \\
\hline P=Promoción & 63,4 \\
\hline F=Formación & 100 \\
\hline I=Infraestructura de apoyo & 31 \\
\hline L=Líneas de investigación & 78 \\
\hline Promedio \% & 68,1 \\
\hline
\end{tabular}

Fuente: elaboración propia.

ción pueden darse por diversos medios, entre ellos la competencia científica y tecnológica, el estímulo intelectual y los incentivos económicos. Los mecanismos de coordinación pueden ser de distinta naturaleza: los consejos de investigación, los institutos nacionales con financiación pública o privada y los grupos internacionales de investigación, todos ellos pueden contribuir a configurar prioridades y estimular la investigación. (2003:5)

Esta cita mantiene vigencia y confirma que es necesario mantener y fortalecer las diversas estrategias para conformar una cultura investigativa universitaria que responda a las expectativas de los actores académicos y a las necesidades sociales.

La tabla 6 y el promedio favorable $(67,2 \%)$ obtenido para la variable cultura investigativa evidencia que tiene debilidades, pero también con fortalezas, como la promoción de la investigación, la gestión de las líneas de investigación y docentes y estudiantes que se asumen como investigadores activos y comprometidos.

Tales resultados alertan acerca de la importancia de fortalecer los actores académicos, ya que de acuerdo con Ramírez, R. (2007) la cultura de la investigación es iniciada por los docentes en forma individual, pero que poco a poco se integran equipos, grupos, comités, centros de investigación y redes que tejen el sistema de investigación en la universidad. Sistema que se desarrolla a través de los procesos educativos, que convergen en el quehacer investigativo en el que intervienen docentes y estudiantes; en este proceso comparten normativas, actitudes, valores, motivaciones, intereses, saberes, experiencias, estrategias, técnicas e instrumentos.

Tabla 6: Variable Cultura Investigativa

\begin{tabular}{|c|c|}
\hline Dimensiones & Promedio Favorable (\%) \\
\hline Actores & 66,3 \\
\hline Estrategias & 68,1 \\
\hline Promedio & 67,2 \\
\hline
\end{tabular}

Fuente: elaboración propia. 


\section{Resultados producto de la interpretación de la información cualitativa}

Los resultados producto de la información obtenida de las entrevistas realizadas en las cibercomunidades de investigación, se categorizaron primero en forma individual, después fueron analizados por grupo y finalmente se integró la categorización general emergente.

Grupo 1 - Docentes: en este caso se integró la opinión de cuatro informantes: directivos, facilitadores, jurados y tutores. En este grupo muchos de los invitados no se integraron a la cibercomunidades de Investigación. Sus categorizaciones se presentan en la figura 1, donde se observa que emergen cuatro categorías con sus respectivas subcategorías. Estas son: investigacion, productos, talento humano y promoción.

En relación con la categoría investigación, la informante 1 afirma que en la universidad se le da mucha relevancia e interés a la investigación, "pondero mucho el interés que se aprecia hacia la investigación" y destacó el aporte de las líneas de investigación como generadoras de publicaciones.

En la categoría productos, emergieron las subcategorías publicaciones y eventos, la informante 2 destaca su importancia como evidencia de una cultura investigativa que está tomando auge y se ha ido robusteciendo, siendo evidencia de ello los órganos divulgativos, así como las jornadas de investigación que se realizan todos los años.

En cuanto a la categoría talento humano, emergieron la subcategoría tutores, en el cual la informante 3 señala que es un trabajo gratificante y sistematizado, y la subcategoría jurado, de los cuales manifiesta que se

Figura 1: Opiniones del grupo docentes

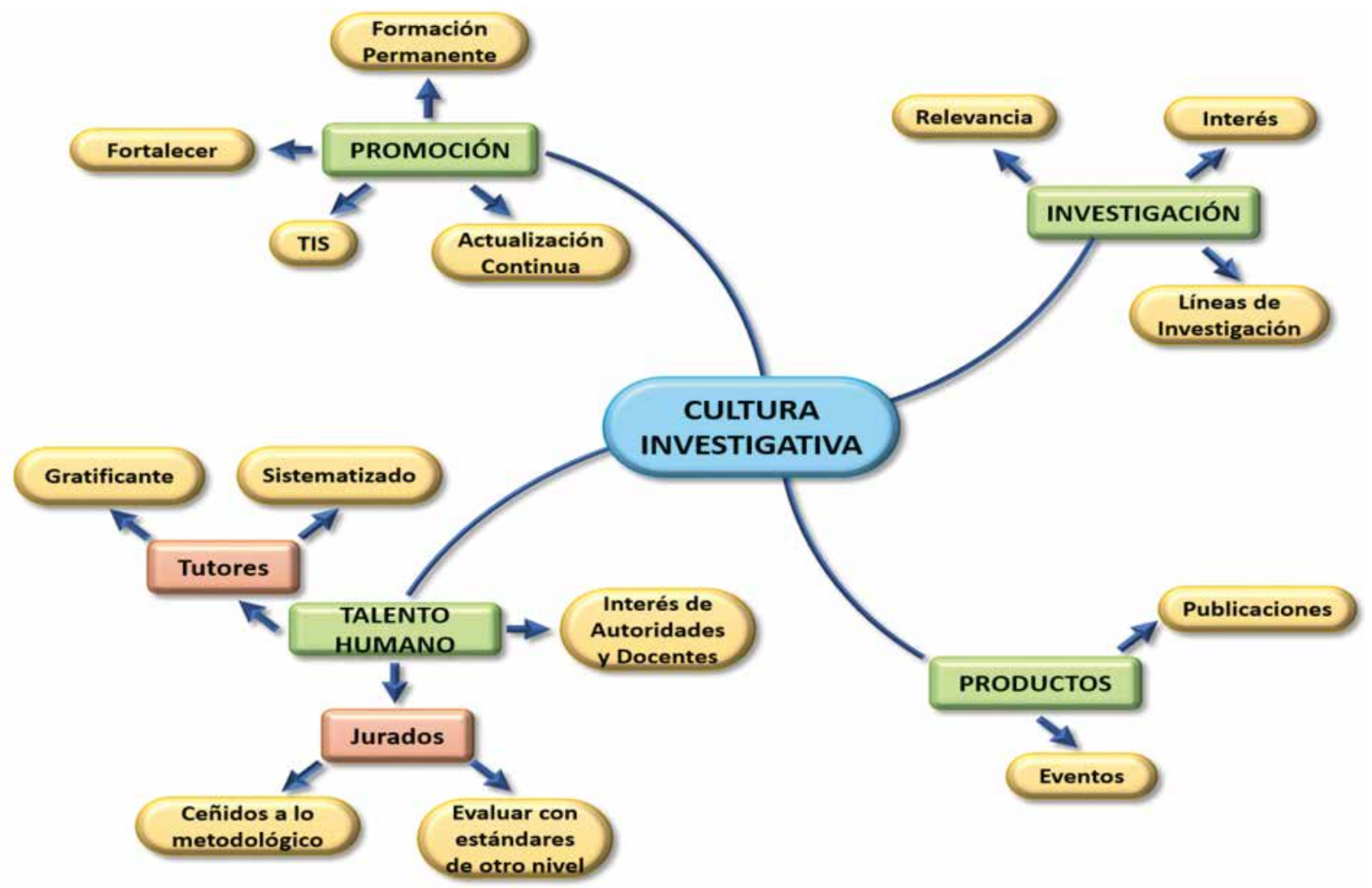

Fuente: elaboración propia. 
mantienen ceñidos a lo metodológico. La misma informante plantea que en algunos casos, cuando estos jurados se encuentran estudiando (por ejemplo el doctorado) y evalúan en maestría, lo hacen con los estándares del nivel superior, o sea en el que ellos estudian, y no con los estándares del nivel al que les corresponde evaluar. Por su parte, la informante 4 (jurado) afirma que es manifiesto el interés de las autoridades y de los docentes en promover la investigación.

En relación a la categoría promoción, la informante 1 afirma que debe mantenerse la actualización continua como se ha venido haciendo hasta ahora, por su parte, la informante 2 hace referencia al Programa de Tutoría Integral Supervisada (TIS) como "un programa exitoso, en tanto un alto porcentaje de participantes logra culminar su trabajo de investigación", señala además que se debe seguir fortaleciendo la cultura de investigación, "porque sin investigación no hay universidad" y mantener la formación permanente de tutores y jurados "a través de talleres y conversatorios".

La matriz de docentes evidencia más aspectos favorables que desfavorables, en la cual tambien los requerimientos están dirigidos al mantenimiento y fortalecimiento de las estrategias existentes, lo que permite inferir que los docentes perciben un ambiente propicio para la cultura investigativa en los estudios de postgrado de la Universidad estudiada. Sin embargo, es importante que la Universidad entre sus politicas de investigación establezca algunos parametros de control en la selección de jurados, que se tienen pero de acuerdo a esto no se cumplen.

Grupo 2 - Estudiantes: la información apor-

Figura 2: Opiniones del grupo Estudiantes

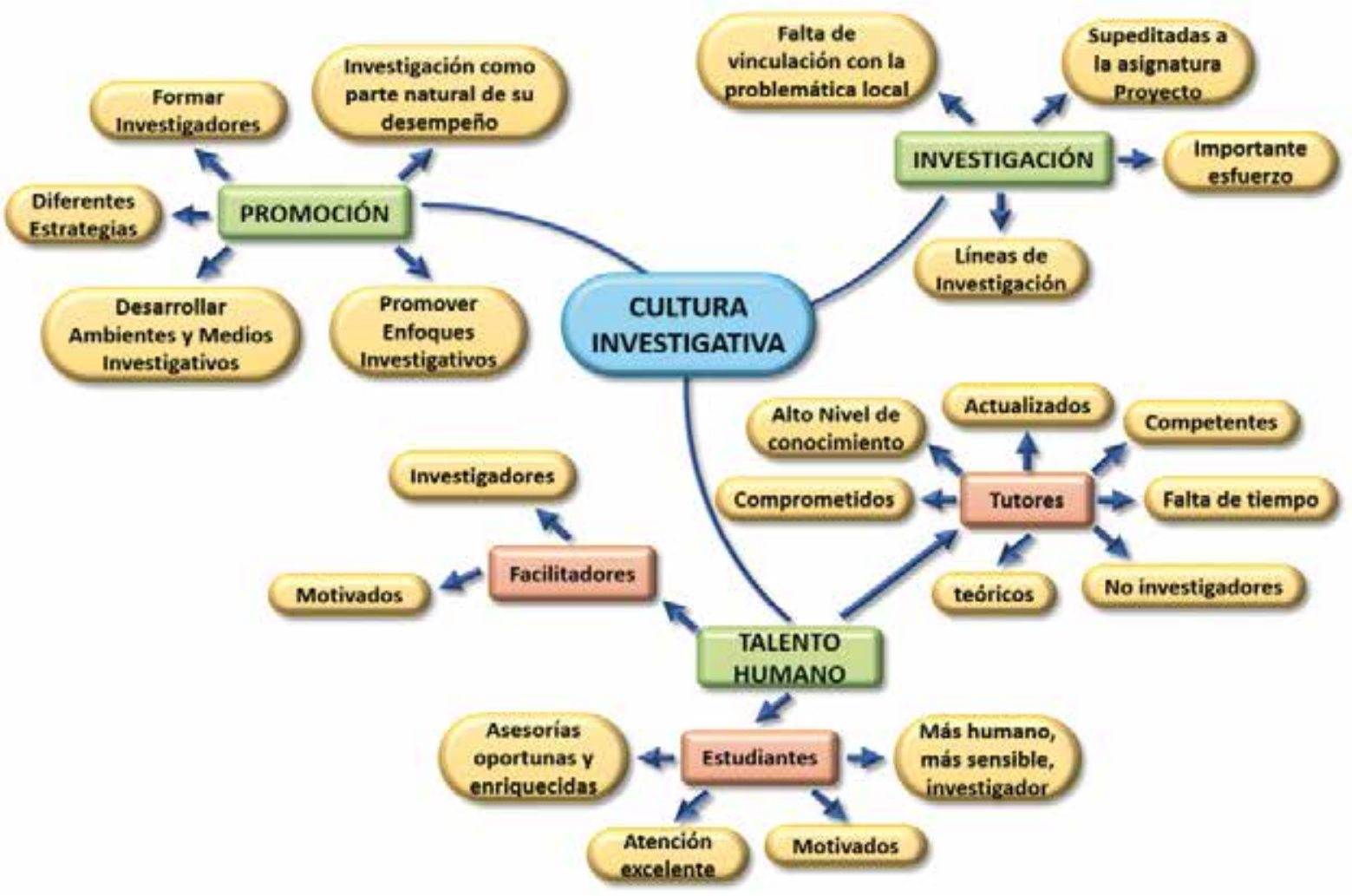

Fuente: elaboración propia. 
tada por los estudiantes en proceso de culminación del postgrado en los programas de Especialización, Maestría y Doctorado, se presentan integradas en las figura 2 , donde se observa que en relación a la cultura investigativa emergen tres categorías: investigación, talento humano y promoción y de cada una, a su vez, emergen varias subcategorías.

En relación con la categoría investigación, el informante 1 manifiesta que falta vinculación con la problemática local: "falta impacto favorable en la sociedad venezolana", por su parte, la informante 2 (tesista doctoral y egresada de Maestría), señala que la investigación se limita a las Unidades Curriculares de Proyecto I y II, para cumplir requisitos académicos: "el proceso investigativo debe formar parte natural de desempeño social, laboral, y personal"; contrariamente el informante 3 opina que se está haciendo un importante esfuerzo: "se cuenta con un robusto programa de cuarto nivel que obliga a los participantes a realizar esfuerzo en cuanto a investigar", lo cual es corroborado por la informante 4 (egresada), quien advierte que las líneas de investigación, coadyuvan a encausar los esfuerzos investigativos.

En la categoría talento humano emergieron varias subcategorías: en cuanto a los tutores, el informante 2 opina que son teóricos, pues "no insertan a los tesistas en investigaciones de verdad", señala además que no son investigadores, por falta de tiempo: "la investigación es un trabajo al que hay que dedicarle, alma, vida y corazón para tener unos resultados modestos", sin embargo, la informante 1 opina que la Universidad cuenta con tutores competentes, el informante 2 cree que están comprometidos y la informante 4 considera que poseen un alto nivel de conocimiento.

En relación a los estudiantes el informante 1 percibe que la experiencia de cursar estudios de postgrado los hará más humanos, más sensible e investigadores. En este orden de ideas, el informante 2 considera que están motivados para investigar y por su parte el informante 3 piensa que reciben por parte de los facilitadores y tutores una atención excelente, lo cual es compartido por el informante 4 quien aprecia que se reciben asesorías oportunas y enriquecedoras.

En cuanto a los facilitadores, los cuatro informantes coinciden en señalar que se encuentran motivados y son investigadores, así la informante 1 expresa "La mayoría de los facilitadores, con los que he compartido cada asignatura, tienen un gran recorrido académico e investigativo".

En la categoría promoción, la informante 2 opina que se requieren implementar estrategias que permitan al tesista ver la investigación como parte natural de su desempeño, para lo cual se deben desarrollar ambientes y medios investigativos para ejecutarlos, pero no describe cuales podrían ser, aplicando diferentes estrategias, que permitan formar investigadores mediante la promoción de nuevos enfoques investigativos; "se trata de generar enfoques investigativos, un enfoque multidisciplinar, bajo paradigmas de la complejidad y la transcomplejidad y revertir las tendencias tradicionales de investigación".

En la matriz de los estudiantes emerge nuevamente el tiempo como elemento desfavorable, lo cual implica que cualquier política investigativa institucional requiere el análisis de cómo sortear este obstáculo y se destaca además como aspectos a mejorar la selección de temáticas investigativas contextualizadas y el patrocinio de tutores con competencias investigativas teórico-prácticas, lo cual implica, que la Universidad debe realizar una evaluación de los tutores que implique el cumplimiento de unas mínimas exigencias y un acompañamiento institucional, a los fines de garantizar 
que responda a las expectativas del estudiante y de la universidad.

La tabla 7 que se presenta a continuación, resume las categorías obtenidas de todos los grupos se actores que participaron en la indagación acerca de la cultura investigativa en la universidad estudiada, clasificadas en

Tabla 7: Categorización General

\begin{tabular}{|c|c|c|c|}
\hline Categorias & Favorable & Desfavorable & Requerimientos \\
\hline Investigación & $\begin{array}{l}\text { Actualización Constante } \\
\text { Diversidad de Criterios } \\
\text { Búsqueda de } \\
\text { Conocimientos } \\
\text { Relevancia } \\
\text { Interés } \\
\text { Línea de Investigación } \\
\text { Importante Esfuerzo }\end{array}$ & $\begin{array}{l}\text { Avance Lento } \\
\text { Poca } \\
\text { Estudiantes Investigadores } \\
\text { Falta de Tiempo }\end{array}$ & \\
\hline Producto & $\begin{array}{l}\text { Publicaciones Periódicas } \\
\text { Nuevos Aportes } \\
\text { Innovaciones } \\
\text { Eventos }\end{array}$ & & \\
\hline Talento Humano & $\begin{array}{l}\text { Profesionales con mística } \\
\text { Preparación } \\
\text { Equipo que aprende } \\
\text { Interés de autoridades y } \\
\text { docentes } \\
\text { Tutores } \\
\text { Trabajo gratificante } \\
\text { Sistematizado } \\
\text { Competentes } \\
\text { Comprometidos } \\
\text { Alto nivel de conocimiento } \\
\text { Tutorías oportunas } \\
\text { Facilitadores } \\
\text { Motivados } \\
\text { Investigadores } \\
\text { Estudiantes } \\
\text { Motivados } \\
\text { Atendidos } \\
\text { Investigadores }\end{array}$ & $\begin{array}{l}\text { Tutores } \\
\text { Poco apoyo } \\
\text { Poca participación } \\
\text { Falta de tiempo } \\
\text { Teóricos } \\
\text { No investigadores } \\
\text { Jurados } \\
\text { Preminencia a lo } \\
\text { metodológico } \\
\text { Evaluación con estándares } \\
\text { de nivel superior }\end{array}$ & $\begin{array}{l}\text { Facilitadores } \\
\text { apropiados del } \\
\text { conocimiento } \\
\text { Grupo de } \\
\text { Investigadores } \\
\text { Apoyo de tutores }\end{array}$ \\
\hline Promoción & $\begin{array}{l}\text { Compartir de saberes } \\
\text { Invitaciones a intercambios } \\
\text { Estrategias } \\
\text { Actualización continua } \\
\text { TIS }\end{array}$ & & $\begin{array}{l}\text { Estímulo al potencial de } \\
\text { los estudiantes } \\
\text { Estímulo al investigador } \\
\text { Tiempo para investigar } \\
\text { Pautas Directivos } \\
\text { Actualización continua } \\
\text { Fortalecimiento cultura } \\
\text { investigación } \\
\text { Formación permanente } \\
\text { Estrategias } \\
\text { Ambientes y medios } \\
\text { investigativos } \\
\text { Formación } \\
\text { investigadores } \\
\text { Promoción de nuevos } \\
\text { enfoques investigativos }\end{array}$ \\
\hline Transcomplejidad & $\begin{array}{l}\text { Pensamiento Crítico } \\
\text { Nueva Forma } \\
\text { Integrador }\end{array}$ & & $\mathrm{TIC}$ \\
\hline
\end{tabular}

Fuente: elaboración propia. 
aspectos favorables, desfavorables y requerimientos.

Este cuadro concentra la información cualitativa obtenida de todos los actores lo cual, permite visualizar con claridad que son más los aspectos favorables de la cultura investigativa en la universidad estudiada, donde destaca como categoría emergente la transcomplejidad, asumida por los actores como pensamiento crítico, como nueva forma de investigar y como enfoque integrador. Tales resultados contrastan con la información cuantitativa donde una sola tesis doctoral asume el referido enfoque.

Por su parte, los aspectos desfavorables se aspiran subsanar con los requerimientos que se concentran en el talento humano, con énfasis en los tutores y en la promoción, con énfasis en la infraestructura de apoyo. Al respecto Parra (2010), manifiesta que para investigar debe existir un alto nivel de motivación y debe girar en torno a los ejes temáticos que se encuentran relacionados con el contexto social, de allí, la necesidad de tomar en consideración la experiencia dentro de la investigación, tanto desde lo individual, del propio ser humano, lo intrínseco, los subjetivo, su propia vida llena de sentimientos, saberes y complejidades, lo que implica replantearse la visión de las cosas y mantener una apertura mental de la diversidad existente, como desde lo social, pues debe guiarse con base al contexto en que se desenvuelve, para ello, se debe tener claro, que cada persona tiene su propio contexto y por tanto, lo que dice una persona es interpretada diferente por otra.

\section{CONCLUSIONES}

A partir del estudio realizado y con base en los resultados se puede concluir que la cultura investigativa en los estudios de postgrado de la universidad estudiada, aún presenta debilidades, de ahí que es necesario enfocar esfuerzos que permitan generar un consenso en cuanto a la apertura investigativa con fundamento en la transcomplejidad como enfoque integrador.

En cuanto a los actores académicos, aunque la opinión generalizada es bastante positiva, es necesario revisar la selección de algunos jurados, puesto que se manifiestan algunas incongruencias, en cuyo caso es necesario el apoyo del tutor y la supervisión de los directivos.

En relación a las estrategias es necesario mantenerlas, reforzarlas y darles mayor difusión, pues en algunas ocasiones se desconocen algunas actividades de interés.

\section{REFERENCIAS BIBLIOGRÁFICAS}

Alfonzo, N. (2015). El Oxímoron del Método sin Método en La Transcomplejidad en la Formación de Investigadores. Ensayos de Investigación, 1(1), 8-15.

Balza, A. (2013). Pensar la Investigación Postdoctoral desde una Perspectiva Transcompleja. San Juan de los Morros, Venezuela: REDIT.

Bonilla-Castro, E. \& Rodríguez, P. (2005). Más allá del Dilema de los Métodos. Bogotá, Colombia: Editorial Norma.

Bracho, K., Molina, G., Carruyo, N. \& Ureña, Y. (2010). Cultura Investigativa: Enfoque Transcomplejo para la Producción Científica en Universidades Modo 2. Maracaibo, Venezuela: LUZ.

González, R. (2007). Investigación Cualitativa y Subjetividad. Los Procesos de Construcción de la Información. México: McGraw Hill. 
Leal, J. (2005). La autonomía del sujeto investigador y la metodología de investigación. Venezuela: UCLA.

Martins, F. (2005). La Interdisiciplinariedad y la Cultura de Investigación del Profesor Universitario en Cosmovisiones de la Educación en el Contexto de la Transcomplejidad. Venezuela: SIPTIC.

Moreno, S. (2005). Diagnóstico de los Estudios de Postgrado. San Joaquín de Turmero, Venezuela: UBA.

Morlés, V., Nuñez, J. \& Alvarez, M. (2010). Transdisciplinariedad, Especialización y Postgrado: Un Debate Necesario. Interdisciplinariedad y Postgrado. Pasado, Presente y Futuro, 9(26), 19-27.

Najmanovich, D. (2001). Epistemología: Una mirada post-positiva. Recuperado de http://www.edupsi.com/epistemologia.htm

Parra, G. (diciembre de 2010). Visiones complejas de la investigación. En j. Divo (Presidente), VII Congreso de Investigación y I Congreso Internacional de Investigación. Universidad de Carabobo, Venezuela.

Ramírez, R. (2007). Concepciones y Modelos de la Cultura Investigativa en Pregrado. Táchira, Venezuela: UPEL.

Ramírez, T. (2007). Como hacer un Proyecto de Investigación. Caracas, Venezuela: Editorial Panapo.

Universidad Bicentenaria de Aragua (2015a). Informe de Gestión de la Dirección de Investigación. San Joaquín de Turmero, Venezuela: UBA-DIEP.

Universidad Bicentenaria de Aragua. (2015b). Consejo Universitario. Resolución No 201-A. Modelo Educativo. Turmero, Venezuela: UBA.

Universidad Bicentenaria de Aragua. (2015). Políticas Investigativas. Turmero, Venezuela: UBA.

Universidad Bicentenaria de Aragua. (2017a). Manual para la Elaboración, Presentación y Evaluación del Trabajo de Grado de los Programas de Postgrado. San Joaquín de Turmero, Venezuela: UBA-DIEP.

Universidad Bicentenaria de Aragua. (2017b). Lineamientos para la Elaboración de Articulas Académicos. Turmero, Venezuela: UBA.

UNESCO. (2003). La Educación Superior en Venezuela. Recuperado de http://unesdoc.unesco.org/images $/ 0013 / 001315 / 131594 s$ s.pdf

Villegas, C., Schavino, N., Rodríguez, J., Moreno, S., Extraño, A., Reyes, N., Salazar, A., Ferrer, L., Diaz, L., Lamardo, G. \& Guzmán, M. (2006). La investigación: Un enfoque integrador transcomplejo. Venezuela: UBA.

Zaa, S. (2015). Tendencias Ontoepistemológicas del Pensamiento Científico Contemporáneo. Filosofía Transcompleja. Otras de Pensar, Ser y Sentir, 1(2), 8-12.
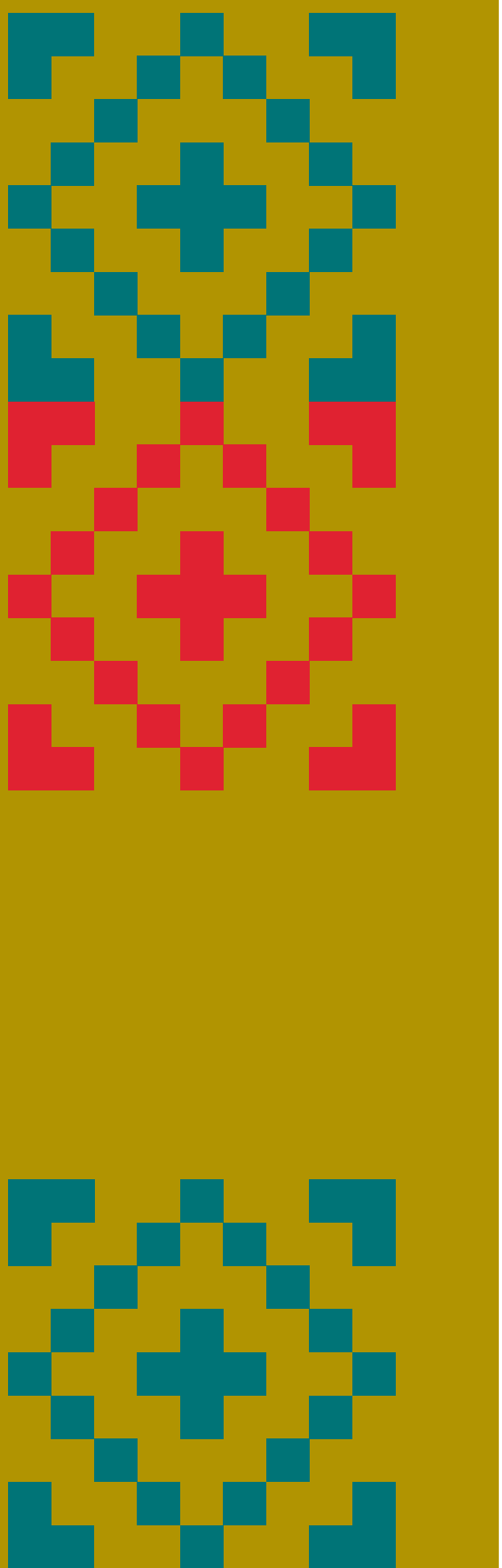